\title{
Probing the Crystallography of Ordered Phases by coupling Orientation Microscopy and Atom Probe Tomography
}

\author{
S. Meher ${ }^{1}$, P.Nandwana $^{1}$, T. Rojhirunsakool ${ }^{1}$, J. Tiley $^{2}$ and R. Banerjee ${ }^{1}$ \\ ${ }^{1}$ Center for Advanced Research and Technology and Department of Materials Science and Engineering, \\ University of North Texas, Denton, TX, U.S.A. \\ ${ }^{2}$ Materials and Manufacturing Directorate, Air Force Research Laboratory, Dayton, OH, USA
}

Superalloys are a class of materials that possess stable microstructures at elevated temperatures due to the presence of coherent $\mathrm{L}_{2}$ ordered $\gamma^{\prime}$ precipitates spatially aligned along elastically soft directions in a face centered cubic (FCC) $\gamma$ matrix [1]. Nickel-base superalloys are used in a variety of applications such as in turbine blades of aircraft engines and land-based turbine engine [1]. The recent discovery of novel Co-base alloys [2] which also form $\gamma-\gamma^{\prime}$ microstructures, similar to nickel base superalloys, has led to rapid research in these alloys as potential next generation superalloys.

The structural and compositional information obtained by APT has given insights to the atomistic behavior of nickel and cobalt-base superalloys [3-4]. The determination of atomic scale structural and compositional information using atom probe tomography is currently limited to elemental solids and dilute alloys. In the present article, a novel reproducible approach has been presented to extract crystallographic information using APT from highly alloyed materials, by synergistic coupling of orientation microscopy with APT, which has been demonstrated for both nickel and cobalt-base superalloys.

The standard protocols like Fourier transforms and Hough transforms have successfully identified crystallographic information in APT in pure or low alloyed systems [5-6]. But it is very difficult to apply these protocols to multi-phase and multi-component systems involving solute elements with high evaporation fields, since multiple poles are not observed in such systems, making their identification rather difficult. The EBSD mediated precise site-specific sample preparation is an alternate method enabling crystallographic study of complex multi-phase materials, containing ordered phases, by APT. The technique proposed in this paper offers the advantage of selecting a desired crystallographic orientation of the material for a lift-out, which enables the evaporation of material with known crystallographic poles. The structural information obtained by APT coupled with other advanced microscopy tools or computational modeling offers new prospects for solving materials science problems. The true nature of atomic architecture in superalloys is being recognized recently, and the advancement presented in the current work with regards to crystallography in APT has immense potential in elucidating the nature of buried interfaces and accurately determining the site occupancy of solute atoms within the ordered precipitates, primarily responsible for strengthening these high temperature materials. Additionally, the underlying physical rationale of field evaporation behavior with respect to type of ordered structure and operating temperature has been addressed in the present study.

\section{References :}

[1] R.C. Reed,The superalloys fundamentals and applications 2006, Cambridge University Press.

[2] J. Sato, T. Omori, K. Oikawa, I. Ohnuma, R. Kainuma, K. Ishida, Science 2006, 312: 90-91.

[3] S. Meher, H-Yan, S. Nag, D. Dye, R. Banerjee, Scr.Mater. 2012, 67: 850 -853.

[4] D.N. Seidman, Annual Review of Materials Research 2007, 37: 127-158. 
[5] B. Gault, M.P. Moody, J.M. Cairney, S.P. Ringer, Materials Today 2012, 15: 378-386.

[6]A.V. Ceguerra, A.J. Breen, L.T. Stephenson, P.J. Felfer, V.J. Araullo-Peters, P.V. Liddicoat, X. Cui, L. Yao, D. Haley, M.P. Moody, B. Gault, J.M. Cairney, S.P. Ringer, Current opinion in solid state and materials science, 2013,17, 224-235

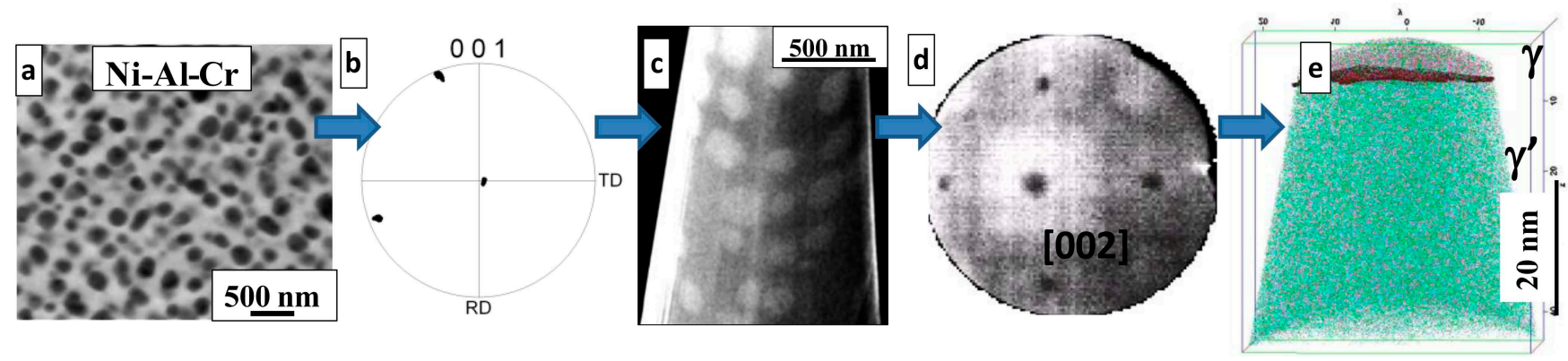

Figure 1. (a) Backscattered SEM micrograph of Ni-14Al-7Cr (at \%) showing $\gamma^{\prime}$ precipitates in a $\gamma$ matrix (b) EBSD pole figure showing the dual-beam FIB lift-out to be closer to [001] axis with an offset of $2^{\circ}$ (c) the pre-final tip prepared using dual-beam FIB shows alignment of precipitates along [001] direction with minimal offset (d) 2D desorption image show poles and zone lines with central [001] pole, having an four-fold symmetry (e) APT reconstruction show a near horizontal $\gamma-\gamma$ ' interface.
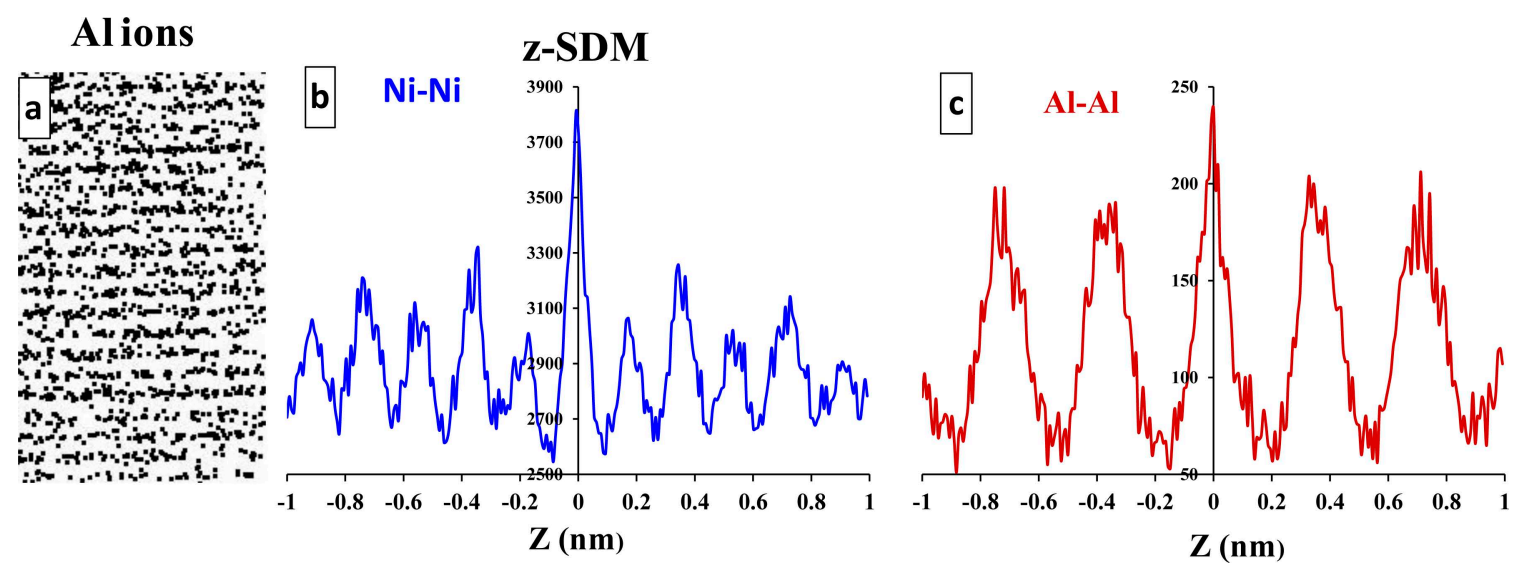

Figure 2(a) Lattice planes of $\mathrm{Al}$ atoms in a region down the [001] pole in a APT reconstruction for Ni14Al-7Cr (at \%) sample (b) z- Spatial distribution map (SDM) for Ni showing lattice planes of Co along [001] direction which are $0.18 \mathrm{~nm}$ widely spaced(c) z- SDM for Al showing lattice planes of Co along [001] direction which are $0.36 \mathrm{~nm}$ widely spaced 